\title{
Pensar las prácticas de los trabajadores sociales en las escuelas desde la construcción adultocéntrica de la juventud. Una lectura posible desde la mirada de Foucault
}

Tatiana Leonor Lescano ${ }^{1}$

\section{Universidad Nacional de Rosario -Universidad Nacional de Mar del Plata- Instituto Superior de Formación Docente y Técnica № 33}

\section{Ensayo}

Material original autorizado para su primera publicación en el Journal de Ciencias Sociales, Revista Académica de la Facultad de Ciencias Sociales de la Universidad de Palermo.

Recepción: 24-02-2019

Aceptación: 28-03-2019

Resumen: El presente trabajo pretende referirse de modo general a la producción de nociones y representaciones de lo juvenil desde el poder adultocéntrico, y marcar algunas entradas para la reflexión de esta problemática a partir del pensamiento de Michel Foucault. A partir de ese punto, pensar el trabajo cotidiano de las y los trabajadores sociales que realizan sus tareas en las escuelas bonaerenses. El objetivo de este escrito es reflexionar sobre el constructo juventud como expresión de las relaciones de poder intergeneracional y sobre cómo la normatividad que los adultos imponen a las nuevas generaciones tiene como finalidad no solo socializarlos, hacerlos parte de lo social, sino también sujetarlos. En una primera parte se explicita la construcción socio histórica de la juventud dentro de una sociedad cambiante, de la que se establecen algunas características, a partir de enunciar un estado de situación sobre lo que hoy se entiende de manera general por juventud y las características de la sociedad en la que esta habita. En la segunda parte se reflexiona sobre la categoría "adultocentrismo", en relación con las normas y la construcción del sujeto joven, y se ponen en tensión los conceptos desarrollados en la primera parte, a partir de cuestionar lo generalmente aceptado. En la tercera parte de este trabajo se realiza una breve reflexión sobre las implicancias de este tema para el cotidiano de los y las Trabajadoras Sociales que trabajan en las escuelas públicas de nivel secundario de la Provincia de Buenos Aires, teniendo en cuenta que toda relación pedagógica lleva implícita el reconocerse y asumirse como una entre dos formas de ser sujeto (adultos y jóvenes) y que la dinámica de las instituciones educativas comprende también una mirada adultocéntrica de los jóvenes.

\footnotetext{
1 Licenciada en Trabajo Social (UNLaM), Especialista en Nuevas Infancias y Juventudes (UNICEN) y Doctoranda en Trabajo Social (UNR, extensión UNMdP). Correo electrónico: lescanotatiana@gmail.com
} 
Palabras clave: juventudes; adultocentrismo; trabajo social; escuela.

\section{Thinking the practices of social workers in schools from the adult-cultural construction of youth. A possible reading from Foucault's gaze}

Abstract: The present work intends to refer in a general way to the production of notions and representations of young people from the power centred on the adult, marking some entries for the reflection of this problem from the thought of Michel Foucault. From this reflection, we invite the thinking about the daily work of the social workers who perform the tasks in the schools of Buenos Aires. The objective of this article is to analyse the construction of youth as the expression of intergenerational power relations, and how the regulations that adults impose on new generations not only make part of the social, but also subject them. In a first part, the sociohistorical construction of youth in a changing society is explained, in which some of the characteristics are presented, in the presentation of a state of the situation in which today youth and characteristics are understood in a general way. Of youth. The society in which he has lived. The second part reflects on the category "adultcentrism", in relation to standards and the construction of meaning. In the third part of this work a brief reflection of the implications of this topic for the daily life of the Social Workers who work in the secondary public schools of the Province of Buenos Aires is made, taking into account that all pedagogical relationship it implies implicitly recognizing and assuming oneself as one between two forms of being subject (adults and young people) and that the dynamics of educational institutions imply an adult-centric view of young people.

Key words: youth; adultcentrism; social work; school.

\section{Introducción}

El presente trabajo pretende referirse de modo general a la producción de nociones y representaciones de lo juvenil desde el poder adultocéntrico, y marcar algunas entradas para la reflexión de esta problemática a partir del pensamiento de Michel Foucault. A partir de esa reflexión, pensar el trabajo cotidiano de las y los trabajadores sociales que realizan sus tareas en las escuelas bonaerenses.

El objetivo de este escrito es reflexionar sobre el constructo juventud como expresión de las relaciones de poder intergeneracional y sobre cómo la normatividad que los adultos imponen a las nuevas generaciones tiene como finalidad no solo socializarlos, hacerlos parte de lo social, sino también sujetarlos.

En una primera parte se explicita la construcción socio histórica de la juventud dentro de una sociedad cambiante, de la que se establecen algunas características, a partir de 
enunciar un estado de situación sobre lo que hoy se entiende de manera general por juventud y las características de la sociedad en la que esta habita.

En la segunda parte se reflexiona sobre la categoría "adultocentrismo", en relación con las normas y la construcción del sujeto joven, y se ponen en tensión los conceptos desarrollados en la primera parte, cuestionando lo generalmente aceptado.

En la tercera parte de este trabajo se realiza una breve reflexión sobre las implicancias de este tema para el cotidiano de los y las Trabajadoras Sociales que trabajan en las escuelas públicas de nivel secundario de la Provincia de Buenos Aires, teniendo en cuenta que toda relación pedagógica lleva implícita el reconocerse y asumirse como una entre dos formas de ser sujeto (adultos y jóvenes) y que la dinámica de las instituciones educativas comprende una mirada adultocéntrica de los jóvenes.

\section{La construcción social e histórica del concepto de juventud: juventudes y sociedad pos moderna}

\subsection{Juventudes}

Margulis y Urresti (1998) definen la juventud como un constructo social, a partir de las categorías de moratoria social y vital. Si bien la categoría joven tiene su anclaje en una situación etaria, (a partir de los 12 o 13 años, cuando empieza la maduración biológica), son la moratoria vital (la distancia desde el nacimiento y la lejanía de la muerte) y la asunción de responsabilidades laborales y familiares, las que delimitan al sector "joven". Estos autores plantean que, al ser una construcción social compleja, es necesario tener en cuenta otras categorías que permitan su análisis, tales como la pertenencia a cierta generación, los procesos culturales de juvenilización, la pertenencia a grupos o "tribus", a una clase social, a un género, y de esta manera poder emprender el abordaje de esta temática.

Los investigadores de la escuela de Birmingham caracterizaron el período histórico en que aparece el concepto de juventud como categoría y actor social en el mundo occidental, considerando las siguientes marcas de la época moderna:

1. La aparición de un mercado, un consumo y una industria orientados a los jóvenes.

2. El incremento de los medios masivos y el nexo entre estos y la cultura juvenil. Es importante aquí la idea de que cada vez más y más personas se ven comprendidas en un proceso de uniformación cultural.

3. El hiato, la grieta de la experiencia social que se precipita por las guerras al inicio del siglo XX. Generalmente los argumentos hablan de los efectos disruptivos de la guerra en los chicos que nacieron en ese período - ausencia de los padres, evacuaciones y otras interrupciones de la vida familiar, violencia- y eso como responsable de la "nueva" 
delincuencia juvenil de los `50, tipificada por los Teds, como los precursores de una tendencia más general de la violencia en la Cultura Juvenil.

4. Cambios en la esfera de la educación, principalmente por dos pivotes de desarrollo. Por un lado "la educación secundaria para todos", que implicó escuelas específicas para este grupo de edad. Antes de la segunda guerra no había escuelas específicas, se recibía la educación secundaria en las escuelas elementales. Este cambio interesa porque crea las condiciones para la emergencia específica de una "sociedad de adolescentes". Por otro lado, la masiva extensión de la educación superior.

5. Último conjunto de cambios, pero no menos importante, es el arribo de un estilo distintivo para todo el grupo, la ropa y la música-rock unen sin ninguna duda a esta generación más joven (Chávez, 2004, p.4).

Aún en la actualidad podemos mencionar como factores estructurantes de la juventud que todavía siguen operando en ella a: la escuela, el mercado y el trabajo, aunque los actores no se ponen de acuerdo sobre cómo o de qué manera lo hacen en el contexto de la sociedad posmoderna.

Dávila caracteriza la juventud urbano-popular como aquella que vaga en un mar de propuestas para la solución de sus necesidades inmediatas, concretas, con una visión pragmáticas de las mismas, y estas en función de un proyecto de vida centrado en la obtención de bienes materiales que la sociedad parece reservar solo para unos pocos privilegiados, "en un símil de carrera que se necesita correr y donde muchas veces los resultados ya se saben, o se tiene perdida antes de correr" (Dávila, 1998, p.82).

Esta juventud, que posee una estatus por debajo del de los adultos, es protagonista de múltiples situaciones de vulneración y subordinación formal frente al mundo de los mayores: a la juventud urbano-popular, proveniente de familias de sectores pobres económicamente, se la identifica como aquella en la cual se registran los índices más altos de fracaso escolar (Balbi, 2005, p.141). Son considerados muchas veces como ciudadanos de segunda clase, o constructores de una ciudadanía de baja intensidad (Kessler, 2004). Y también portadores de estigmas sociales por su condición de edad (juventud), raza, género; y que viven en escenarios de violencias simbólicas y discriminación simplemente por ser jóvenes. Los que además de jóvenes son pobres transitan por dos niveles de exclusión: la primera, por ser joven en un mundo de adultos; la segunda, la de la pobreza de los sectores urbanos.

En Argentina, existe además un tercer nivel de exclusión que implica vivir en un país de Latinoamérica en donde el Estado, durante muchos años, se ha retirado de la esfera social y ha limitado considerablemente las garantías que ofrece a la población. En el decenio 2005 
- 2015 se han implementado políticas públicas que intentaron crear condiciones que permitan garantizar el derecho a la educación para todos los habitantes y mejorado las posibilidades de inclusión social, aunque parecen revertirse a partir del 2015.

Todas estas condiciones prefiguran una categoría juventud que es heterogénea. Es un error considerar a todos los jóvenes por igual como categoría homogénea, y es por eso que se elige hablar de juventudes, utilizando el plural del término. Esta pluralidad, y la conciencia de la misma, ha sido muchas veces la base para las luchas por las reivindicaciones sociales, sobre todo en aquellos aspectos más sensibles de la exclusión: la discapacidad, la diversidad sexual, el derecho a poder mantener una identidad diferente sin que tenga como consecuencia la discriminación (Del Río Lugo, 2004, p.20). Como dice Dagnino, "todo campo político relevante es siempre un campo minado, un campo de disputa por la fijación de significados. Los mecanismos de apropiación y desapropiación de significados son parte constitutiva de la lucha política" (Dagnino, 1994).

\subsection{La sociedad que habitan los jóvenes. La metamorfosis de la cuestión social: debilitamiento institucional y la modificación de la relación entre sociedad y sujeto}

Así pensada, la categoría de juventudes es entendida en relación con la sociedad de la que forma parte, por lo que se hace necesario realizar el esfuerzo de comprender la complejidad de la sociedad contemporánea.

Diferentes autores han acuñado diversas categorías para explicar las transformaciones estructurales. Castells (1998) habla de sociedad de red; Giddens (1995) de modernidad reflexiva; Beck (1997) de sociedad de riesgo; y Luhmann (1998) de sociedad mundial. Más allá de los nombres elegidos, debemos tener en cuenta que todos coinciden en sus conceptualizaciones al señalar que se está produciendo una reestructuración de las relaciones sociales en relación con la histórica modernidad occidental, así como de los marcos regulatorios de la acción de los individuos e instituciones.

Se ha dado un quiebre en la matriz estado céntrica (Cavarozzi, 1999) características de los Estados modernos occidentales, lo que desplaza al Estado como eje de la integración social y como actor principal en el control y administración de la vida social. Además del quiebre de esta matriz, nuestras sociedades se encuentran atravesadas por procesos que algunos autores denominan de desinstitucionalización, cuestionando la efectividad de las instituciones tradicionales en los procesos de subjetivación, socialización e individuación. 
En este sentido, no solo el Estado, sino todas las instituciones que constituyen los marcos que regulan y contienen la existencia de los individuos, explotan: la familia, la escuela, el Estado, la fábrica, las organizaciones de representación social y política.

Asistimos entonces a un desencaje (Giddens, 1995), en donde la inexistencia o transformación de las condiciones de la sociedad industrial provocan una descomposición del entramado de experiencias que regula la existencia de los individuos que están condenados a ser ellos mismos (Sennett, 1978).

Las relaciones entre individuo y sociedad se ven modificadas. Tanto Beck como Giddens acuñan el término "modernización reflexiva", que supone una radical revisión de la dialéctica individuo - sociedad, y el de "individualización", que presupone al "individuo como actor, diseñador, malabarista y director de escena de su propia biografía, identidad, redes sociales, compromisos y convicciones. O sea, significa la desintegración de las certezas de la sociedad industrial y la compulsión por encontrar las propias. Castell, además, suma a esto lo que llama el proceso de "desindividualización", en tanto los individuos se ven articulados con las colectividades constituidas sobre la base de la división del trabajo, construyendo su identidad a partir de un colectivo alienante.

Pensadores latinoamericanos como Robles (1999) señalan que los modelos de la construcción de la individualidad, así como los paradigmas de reconstrucción, interpretación y observación de la sociología, no pueden ser idénticos en las sociedades del capitalismo desarrollado y en las sociedades de la periferia, estableciendo las diferencias entre los procesos de individuación e individualización.

El primero, el proceso que denomina de individuación, estaría caracterizado como el proceso de construcción de la individualidad que se da en una situación histórica específica, como lo es la pertenencia a la sociedad de riesgo del capitalismo periférico, caracterizada por la masificación y la generalización de la exclusión. Por lo tanto, se daría de manera distinta, distintiva en cada sociedad, y de manera más frágil en aquellas en las que el Estado o las diversas instituciones no operan como sostén de los procesos de socialización de los individuos. Es en este sentido que no se darían de igual manera los procesos de individuación en los países emergentes, pobres, como se dan los procesos de individualización en los países centrales occidentales.

El debilitamiento del entramado institucional propio de la sociedad industrial, en el que se ubica la baja sintonía simbólica del Estado, junto con los procesos de individuación a los que se refiere Robles, abre el interrogante respecto al sistema disciplinar de este nuevo orden social, y al sistema escolar como exponente del mismo (Foucault, 1996). Para Deleuze (1991) atravesamos una crisis generalizada de todos los lugares de encierro. Son los interiores familiares, escolares, profesionales, etc., los que están en crisis. Los encierros son moldes, 
módulos distintos; los controles son modulaciones que cambian continuamente como un tamiz cuya malla cambiaría de un punto a otro.

\subsection{La sociedad moderna y capitalista}

Esta sociedad moderna se constituye además en relación con el proyecto del capitalismo, por lo que es necesario analizar este proceso de entramado del capitalismo dentro del proyecto de la modernidad. Según Boaventura de Sousa Santos (1998), la modernidad que surge en Europa a partir del siglo XVI se constituye como paradigma cultural. Luego, a partir del siglo XVII se instala el capitalismo como modo de producción, entendiéndose no solo como un sistema mercantil sino como un sistema de relaciones entre el capital y trabajo, en donde el segundo queda subordinado al primero. Este sistema de producción capitalista se va transformando en el modo de producción dominante, imbricándose estrechamente con el paradigma cultural de la modernidad, logrando que a partir de mitad del siglo XIX formen una unidad difícil de distinguir, incluso analíticamente.

Los dos pilares que sustentaron el proyecto de la modernidad fueron: el de la regulación y el de la emancipación.

- Regulación (principios que la sustentan):

- Principio del Estado (analizado por Hobbes)

- Principio del Mercado (sustentado por Locke)

- Principio de Comunidad (sustentado por Rousseau)

- Emancipación (articula tres dimensiones de racionalización y secularización de la vida colectiva):

- La Racionalidad moral práctica (basada en el derecho moderno)

- La Racionalidad cognitivo experimental de las ciencias y las técnicas modernas

- La Racionalidad estético - expresiva de las artes y de la literatura moderna

El equilibrio entre estos pilares fija una meta positivista de "orden y progreso" para la sociedad. Los desequilibrios generados entre ambos pilares, así como las relaciones contradictorias entre sus diversas dimensiones, complejizan lo que se conoce como la "cuestión social".

El desequilibrio en el pilar de la regulación consistió globalmente en el desarrollo hipertrofiado del principio del mercado en detrimento del principio del Estado y de ambos en detrimento del principio de la comunidad. Se trata de un proceso histórico no lineal que en las sociedades capitalistas avanzadas, incluye una fase inicial de hipertrofia total del mercado, en el período del capitalismo liberal; una segunda fase, de mayor equilibrio entre el principio del mercado y el principio del Estado bajo la presión del principio de la comunidad, el período del capitalismo organizado y su forma política propia (el Estado-Providencia); y por último, una fase de re-hegemonización 
del principio del mercado y de colonización por parte de éste, del principio del Estado y del principio de la comunidad... (Sousa Santos, 1998, p. 287).

Laval y Dardot (2012) denuncian a su vez a un mercado omnisciente, omnipotente y autorregulador. Demuestran que este aparente caos procede de una racionalidad cuya acción es subterránea, difusa y global. Dicha lógica construye y define la esencia del neoliberalismo. Al explorar su génesis doctrinal, los autores despejan numerosos malentendidos: no se trata de un retorno al liberalismo clásico, ni un retorno a un capitalismo «puro», y sostener este contrasentido es no entender sus nuevos rasgos. Por múltiples vías, el neoliberalismo se ha impuesto como la nueva razón del mundo, que hace de la competencia la norma universal de los comportamientos, sin dejar intacta ninguna esfera de la existencia humana. Una lógica tan corrosiva erosiona hasta la concepción clásica de la democracia, e introduce formas novedosas de sujeción que constituyen, para quienes las rechazan, un desafío político e intelectual inédito. Los jóvenes no escapan a estas lógicas.

Es importante, por tanto, explicitar los cambios que vienen sucediendo en la estructura social, ya que, siguiendo los dichos de Castro (2005), los jóvenes constituyen un colectivo socio-generacional cuyas características de identificación como tales se hallan estrechamente vinculadas con aspectos del tiempo socio-histórico. Desde aquellos estudios sobre juventud que se realizaron en la etapa de la posguerra hasta los que actualmente se vienen desarrollando, es posible observar la influencia de elementos contextuales en los modos de describir a los jóvenes. El enfoque de análisis que deviene de los estudios culturales aporta una particular mirada para el conocimiento del colectivo juvenil al incorporar la influencia del contexto social en el análisis del tema.

Considerar entonces a las Juventudes como un constructo social, histórico y cultural implica poner la mirada en la sociedad de la que es parte, pero de una manera relacional. En este sentido, la pregunta es ¿quiénes construyen a este nuevo actor social juventud? y ¿qué relaciones de poder se establecen a partir de esta construcción?

\section{La mirada adultocéntrica: normatividad y subjetivación}

\subsection{La mirada adulta y las características de la juventud}

Uno de los ejes de análisis de Foucault es el poder, visibilizando la ambivalencia de la modernidad al decir:

que hombres dominen a otros hombres, y es así como nace la diferenciación de los valores; que unas clases dominen a otras, y es así como nace la idea de libertad; que hombres se apropien de las cosas que necesiten para vivir, que les impongan una duración que no tienen o que las asimilen por la fuerza, y tiene lugar el nacimiento de la lógica. (Foucault, 1992, p.15) 
La dominación y la irracionalidad han sido dos marcas de nacimiento de la modernidad, que son heredadas por la posmodernidad actual.

Desde esta perspectiva, se entiende que la construcción de los sujetos sociales es resultado de las relaciones de poder que se dan en el interior de las sociedades. La pregunta por el sujeto, en perspectiva de MF, remite necesariamente a la combinación de los niveles arqueológicos, genealógicos y de subjetivación en el marco de las relaciones de poder que han constituido y constituyen la modernidad. "Mi objetivo ha sido crear una historia de los diferentes modos por los cuales, en nuestra cultura, los seres humanos se convierten en sujetos" (Foucault, 2015, p. 31).

Tanto Bordieu como Foucault asumen que las edades, los colectivos etarios en las sociedades, son construcciones sociales. Bordieu afirma que tanto los jóvenes (y la categoría de juventud) y los viejos (y la categoría de vejez) constituyen categorías que no están dadas sino que se construyen socialmente, al expresar que "la edad es un dato manipulado y manipulable, muestra que el hecho de hablar de jóvenes como unidad social, de un grupo constituido, que posee intereses comunes, y referir estos intereses a una edad definida biológicamente, constituye en sí una manipulación evidente..." (Bourdieu, 1990, pp. 164-165).

Al considerar la juventud como una construcción social, se debe considerar la construcción colectiva que se realiza en tanto representaciones y discursos que van moldeando la visión estereotipada sobre los jóvenes. Entonces se piensa la juventud:

- Como etapa vital, entre la niñez y la madurez

- Como etapa de preparación para el mundo adulto (universidad, trabajo, familia)

Ambos enunciados implican que la juventud es una etapa de tránsito que adquiere valor en relación con el mundo adulto, y que su importancia está puesta en que en algún momento se llegará a ser adulto.

Esto impide de hecho valorar la juventud en sí misma, construir parámetros de análisis propios de la juventud y no en relación con otras etapas vitales (no es niñez, no es adultez). Esta mirada desde el mundo adulto, esta construcción de saberes sobre la juventud desde la mirada adulta, es a la que se denomina adultocentrismo.

En este sentido, se puede asumir un análisis de la juventud a partir de los ejes planteados por MF en el análisis de la sexualidad (2009), referido a las visiones biologisistas y evolucionistas sobre la juventud. Estos discursos naturalizan la existencia de la juventud como sujeto, caracterizándolos como grupo homogéneo y desde el déficit:

- Déficit de razón (déficit sustancial)

- Déficit de madurez (déficit cognitivo - evolutivo)

- Déficit de responsabilidad y seriedad (déficit moral) 
- Naturaleza violenta o "revolucionaria" (que opera sobre los estereotipos políticos que implican la juventud).

Este saber ha sido sostenido desde la psicología evolutiva (por ejemplo en Erikson, 1997) y ha sido aceptado como un hecho "científico", casi un dogma, y permeado las prácticas escolares sin ser cuestionado. Este discurso de "verdad" sobre los jóvenes ha jugado como "saber autorizado". En términos de MF (1999), sabemos que la verdad no es producto del ejercicio de la razón sino el resultado de una "voluntad" que la ha producido. A partir de esta verdad, se institucionalizan discursos, convergen disciplinas, se interviene en la corporalidad y, a través de la normativa, se construyen subjetividades.

También los conceptos de moratoria vital y social que se mencionaban al inicio como características de la juventud tienen como consecuencia:

- Homogeneización de todas las personas jóvenes según la edad

- Homogeneización de todas las personas jóvenes en tanto se esperan los mismos comportamientos, sin detenerse a pensar en necesidades, intereses y formas de comprender el mundo

Otra forma de poder adultocéntrico que opera sobre la juventud, en términos de MF, refiere al modo en que funcionan una serie de prácticas históricas. Esta racionalidad pretende convertir en objeto a la realidad humana, de tal modo que hace de la juventud una cosa que puede ser medida (a través de las estadísticas), manipulada (aplicando el mismo código moral para la sanción de los actos) y controlada, ya que no se la reconoce como un interlocutor válido, un sujeto. Esto, en el ámbito escolar, impregnó las formas de lo escolar a partir de las normas disciplinarias, las estadísticas oficiales, las regulaciones, la forma de organizar los tiempos, los espacios y los cuerpos. Según Santiago Castro - Gómez, la población se transforma de un conjunto de personas a un conjunto de procesos y se establece la necesidad de conocer estos procesos para poder gobernarlos y generar las técnicas específicas que le permitan hacer (la "recta disposición de las cosas") (Castro Gómez, 2010, p. 61). En este sentido "el poder adultocéntrico es un poder sobre la vida, mientras que el término juventud se refiere a una población (un proceso, un grupo etario, una estadística)" (Vásquez, 2013, p.225).

En el ámbito escolar encontramos diversos discursos, diversas disciplinas científicas, que estudian la juventud desde miradas biologicistas, psicologistas, sociológicas, culturales, comunicacionales, etc. Para poder realizar un análisis del adultocentrismo en las prácticas escolares de las escuelas de la sociedad moderna, y que este análisis pretendiera tener un carácter instituyente sobre las prácticas que operan sobre las juventudes, hay que plantear la crítica desde los significados que, a su vez, son resultados de invisibilizaciones violentas. 
En términos de MF, se trata de "borrar finalmente la soberanía del significante" (Foucault, 1999, p.51).

Por tanto la pregunta sería ¿cuál es la trama de poderes que se encuentra en la génesis de las "verdades científicas" sobre la juventud? ¿De qué manera estas verdades constituyen simbólicamente al sujeto moderno? ¿Y cómo estas verdades sustentan prácticas que reproducen esta construcción? ¿De qué modos estas prácticas operan como colonizadores de las formas de pensar y de hacer?

Es muy interesante pensar el poder como colonizador, en tanto se apropia de prácticas y sentidos, y otorga otros propios de quienes ejercen el poder, invisibilizando el proceso y naturalizando las consecuencias. Santiago Castro Gómez (2007), en su trabajo Michel Foucault y la colonialidad del poder, explica las formas en que se da la colonialidad del poder a través de estructuras complejas (heterarquía) en las que no existe un nivel básico que gobierne a todos los demás, sino que todos los niveles ejercen alguna forma de influencia mutua en eventos particulares y coyunturas específicas (Castro Gomez, 2007, pp.170-171). Es por esto que pensar la colonialidad implica problematizar lo que "es" (problematizar el ser). A partir de esto podemos pensar que, en esta sociedad moderna, el ser adulto ha operado de acuerdo a la generación de un centro de poder que ha mirado a los jóvenes como los "otros", los bárbaros, en la que medida que ha atribuido a las personas jóvenes unos determinaos rasgos que los han desplazado a la periferia. Esto explica por qué los jóvenes (en la periferia del poder) son caracterizados por los adultos (en el centro del poder) como:

- Los jóvenes son rebeldes por naturaleza

- Los jóvenes son inestables porque esa es su naturaleza

- Los jóvenes son inmaduros, esa es su naturaleza

Esta mirada adultocéntrica, tal lo destaca Vásquez (Óp. Cit.), tiene un carácter esencialista, similar al discurso racial, ya que este tipo de enunciados puede contribuir a la construcción de los "regímenes de verdad" enunciados por MF. En esta verdad construida, los adultos, los mismos adultos serían en su calidad de portadores de "adultez", los que tiene de manera privativa la capacidad de "razón sensata".

\subsection{Los tipos de normatividad que han regulado las prácticas adultocéntricas}

El adultocentrismo debe pensarse en un entramado de relaciones y no como una característica intrínseca de los adultos y como tal opera:

- Simultáneamente

- En distintas intensidades

- En direcciones variables 
Son estas operaciones las que lo configura como heterárquico. Foucault recurrió al análisis de las instituciones educativas (2002) para mostrar las formas de articulación del poder disciplinario. Se puede afirmar que la escuela se ha formado adultocéntricamente, y esto se evidencia en algunas frases, tales como que los jóvenes van a la escuela "para ser alguien en la vida", o que la escuela forma a los jóvenes para que cuando egresen puedan "ser parte del mundo". En la escuela se sostiene la autoridad vertical de los y las docentes y se niega el reconocimiento que constituye como tales al sujeto docente y al sujeto estudiante en una relación pedagógica.

Si pensamos en que la pedagogía se funda en la relación entre dos sujetos que se reconocen como tales, el desarrollo de un pensamiento crítico pasaría por hacer visible el rasgo moderno - colonial por el cual la construcción adultocéntrica de la juventud y la escuela niegan la posibilidad de que los jóvenes sean pensados como sujetos plenos en sí mismos. Pero no solo en el pensamiento, sino en la crítica de las prácticas, es en donde se pone al docente en el lugar de sabio y al estudiante en el lugar del ignorante. La crítica a las propias prácticas educativas, como momento de la praxis de la desnaturalización, visibilización, es el primer paso para considerar a la pedagogía en su poder transformador de lo cotidiano, y de esta manera hacer que las aulas no se conviertan en meras reproductoras del poder societal.

La escuela de la modernidad que heredamos en esta posmodernidad opera con una lógica de "normalización". En una determinada cantidad de años, el niño / joven inacabado es transformado en un ser acabado listo para "ser alguien en la vida". De ahí que se desprenda que, durante los años de formación, su subjetividad se transforma en un objeto que puede ser dominado a partir de que tanto adultos como jóvenes operan en un determinado régimen de verdad, en tanto integra discursos, instituciones, disciplinas y prácticas.

Esta normalización en la que maniobran las escuelas tiene por lo menos dos finalidades: un poder disciplinario, tal como MF lo describe para las escuelas cristianas del siglo XVII y XVIII; y el poder sobre las juventudes contemporáneas desde la concepción de la población, en tanto regulador de la forma en la que se construyen las identidades modernas. De esta manera, se entiende la escuela como un elemento más a través del que opera el adultocentrismo en la dimensión biopolítica, en tanto se establece la diferencia entre normal - anormal a partir de la adecuación o no adecuación a la norma preestablecida (de conducta, de aprendizaje, de apropiación de los diseños curriculares, entre otras).

Pensar en cambiar la mirada adultocéntrica implica que debemos combinar la reflexión sobre la producción de discursos y los mecanismos por los cuales estos discursos circulan y se reproducen. Implica tomar el desafío de trabajar pedagógicamente las violencias que significan los regímenes de verdad legitimados. Implica desprendernos de un 
adultocentrismo que describe el conflicto generacional exclusivamente desde los malestares culturales (drogadicción, alcoholismo, etc.), para centrar el debate en una lectura crítica y dialogada de las prácticas de las personas jóvenes como vía para entender diferentes formas culturales que pueden hacer más accesible la complejidad de este tiempo.

Los tiempos actuales demandan que una nueva forma de producir el discurso sobre los jóvenes no desconozcan a estos sujetos como co-autores del relato. En este sentido estamos hablando de una producción dinámica y de creación de textos asignificantes que tengan como principal interés descubrir cuál es la función que el texto tiene para el lector que acude a leer; dicho en otras palabras, combinar la manera cómo se produce la reflexión acerca de las personas jóvenes con el agenciamiento que suscitan. (Vázquez, 2011, p. 82)

\section{El trabajo social escolar en la trama de las relaciones adultos - jóvenes en el espacio escolar}

Es en esta trama, escolar y adulta, en las escuelas modernas de esta sociedad posmoderna, en la que se desenvuelven los equipos de orientación escolar en las escuelas de la Provincia de Buenos Aires en Argentina. Los Equipos de Orientación Escolar de la Provincia de Buenos Aires están conformados por una cantidad variable de entre 2 y 4 miembros que reciben los nombres de: Orientadores Educacionales (OE - principalmente psicopedagogos / psicólogos); orientadores de los aprendizajes (OA - principalmente maestras acompañantes de las trayectorias); Fonoaudiólogas (FO) y Orientadoras Sociales (OS - principalmente trabajadores Sociales). Las escuelas secundarias (las que reciben a estudiantes entre los 12 y 18 años) cuentan con equipos conformados por dos miembros (OE y OS). Los trabajadores sociales son parte de estos equipos, y ante ellos se van presentando los objetos y situaciones de trabajo a partir de las distintas demandas: las de la institución, la de la organización, la de las personas, las de los profesionales y la profesión.

Los profesionales trabajadores sociales y psicopedagogos, que son parte de estos equipos, no escapan a las lógicas antes mencionadas, respecto al carácter disciplinador, normativo y reproductor de un "régimen de verdad" sobre la juventud, construida desde una mirada adultocéntrica. Sin embargo, la misma institución configura para los equipos de orientación escolar en general, y a los trabajadores sociales en particular, un lugar de trabajo que permitiría pensar en algunas posibilidades para trabajar pedagógicamente la crítica a este sistema y empezar a construir otros sentidos y discursos sobre la juventud. Una de las formas es habilitar posibilidades de intervención a partir de nuevos marcos orientadores, dados por los documentos de trabajo, que dejan en mano de los profesionales un margen de libertad de acción que podría ser utilizado para desnaturalizar prácticas y discursos y construir otros. 
Entre los documentos de trabajo que sirven de marco normativo y que pautan el marco referencia laboral de estos equipos, existe un documento del Ministerio de Educación de la Nación (MEN). Este documento MEN invita a “...delimitar esa posición de quien interviene, reconocer aquello que le da especificidad y al mismo tiempo, describir la trama en la que esa posición se sostiene, cómo, con quiénes y cuándo se configuran el o los objetos de trabajo" (MEN, 2014, p. 22)

Sobre los objetos de trabajo, sostiene que cada uno de estos objetos con que tienen que trabajar los equipos de orientación escolar es no fijo, que debe construirse y contextualizarse; que esta construcción

utiliza materiales que provienen de experiencias diversas del pensamiento sobre esas experiencias, relacionadas con saberes particulares. El pensamiento requiere objetos para detenerse y delimitar un espacio. A su vez, genera sujetos que piensan (MEN, 2014, p. 27).

El objeto de trabajo de un equipo se configura, no como un sujeto, sino como un trabajo de composición, de mediación, tanto material como simbólica. Pensar al objeto de trabajo como composición, demanda la capacidad narrativa para dar cuenta de uno mismo, de su responsabilidad como profesional y de su sustento técnico operativo para realizarlo.

En este punto es necesario pensar hasta dónde un profesional que es parte de un equipo de orientación en una escuela puede "construir" su objeto de trabajo. Aparecen aquí tensiones entre sentidos instituidos y sentidos instituyentes del trabajo profesional, teniendo en cuenta no solo la lógica de la profesión misma de los trabajadores sociales (ts), sino del entramado lógico que sostiene a la escuela en general, que transita entre siglos de tradición y la necesidad de acomodarse, de pensarse, y dar respuestas a nuevas demandas de nuevos actores.

Al pensar la construcción del "objeto" con el que trabajan los trabadores sociales, la Dra. Alicia González Saibene indica que el profesional trabajador social, así como los profesionales que comparten el espacio de trabajo, no pueden configurar el objeto ya que “...está establecido por y en ese espacio de relaciones de poder que configura cada organización" (Saibene, 2007, p. 34). La Dra. Saibene indica que el objeto es una construcción propia de la organización en la que se enmarca el trabajo profesional, y producto síntesis de las historias, las luchas de poderes, los sentidos construidos a través de procesos que se presentan signados por el conflicto, generalmente contradictorios, simbólicamente estructurados y que hacen a la legitimación y consolidación de la institución / organización, en este caso nada más y nada menos que la escuela.

En un primer momento, entonces, parecería haber una contradicción entre la posibilidad de construcción del objeto, que implica en realidad re-pensar a los sujetos, con 
los que trabajan los trabajadores sociales y sus equipos, y la realidad de los objetos de trabajo prefigurados por la institución de pertenencia de estos profesionales. En este sentido, se entiende la construcción de este objeto inmerso en el proceso dinámico y de lucha de poderes que significa la construcción del campo profesional, en donde la norma, en este caso la nombrada del Ministerio de Educación de la Nación, delinea el objeto y, al mismo tiempo, establece estrategias para su abordaje, de manera explícita, mediante programas y proyectos.

Esta relación, caracterizada por tensiones, entre el "objeto organizacional", configurado por la institución/ organización escuela, que es una manifestación singular de un contexto conflictivo social particular, y el "objeto profesional", que se identifica de diversas maneras de acuerda con el posicionamiento teórico - epistemológico- ético que asuma el profesional, se refleja en las relaciones sociales que se estructuran entre sujetos, en función de las necesidades y el contexto social particular y general, y configuran las formas concretas del abordaje de las problemáticas de los equipos de orientación escolar.

Es importante entonces volver a la definición de trama y al discurso que estructura la práctica profesional, pensando que las relaciones de poder que se juegan en el campo institucional escolar van imponiendo su interpretación de los hechos, van construyendo un discurso hasta imponerse con fuerza de verdad. Es entonces a nivel del discurso en donde deben darse las "peleas" por la construcción del objeto, a partir de establecer las definiciones conceptuales que lo configuran y revisitar las prácticas para desnaturalizar las mismas.

El Trabajo Social como profesión es interpretada como una profesión que intenta establecer cambios a partir de sus prácticas que impliquen una mejora de la situación sobre la que se interviene. Sin la posibilidad de revisar los discursos y las prácticas, las construcciones adultocéntricas sobre la juventud se imponen con fuerza de verdad sobre el conjunto de lo escolar en su calidad de "poder pastoral", el cual es ejercido en el Estado Moderno por diversas profesiones que se dedican a "ver sin ser mirados".

Uno podría preguntarse entonces, en términos de Foucault y su "poder pastoral del Estado", si los trabajadores sociales somos o no parte de la maquinaria que este Estado instituye para poder ejercer el dominio e imponer su verdad, y reproducir su ideología.

Si acordamos con el postulado foucaultiano de que el conocimiento es guerra, de que la estructura de la sociedad es estrategia, que no hay sujetos, sino luchas, y que en este campo de batalla por el dominio de la verdad se encuentran múltiples agentes, factores de poder en lucha tratando de imponerse sobre los otros, es necesario que los trabajadores sociales, agentes del Estado, nos preguntemos desde nuestra profesión, cómo actuamos políticamente, en medio de una multiplicidad de hechos que no se pueden entender en su totalidad. Así se concluye que para actuar políticamente es necesario entender. 
"Consideramos que las teorías o enunciados que postulamos para orientar nuestras intervenciones son definitorias para que el derecho a la educación se haga efectivo" (MEN, 2014, p. 10)

¿Cómo lograr la comprensión de este campo tensional que se presenta caótico, que presenta a la historia como fragmentada tal como la concibe Foucault? Es necesario realizar ciertas síntesis para poder actuar: "reflexionar sobre la condición de época... no como fatalidad sino como potenciadora y posibilitadora de nuevos pensamientos, posicionamientos y prácticas profesionales" (MEN, 2014, p. 12)

Lamentablemente, Foucault muere cuando empezaba a pensar en los mecanismos para pensar este poder y las estrategias para realizar acciones instituyentes a ese poder. Empezaba a desarrollar el término "contraconductas" de los sometidos al poder (Kamal, 2014).

Los trabajadores sociales tenemos algunas posibilidades de revisar nuestras prácticas. Una de ellas es repensando la demanda, tanto institucional como profesional, desarmando y rearmando el sentido de la misma desde múltiples miradas. Esta intervención repensada y resituada en la intertextualidad de las miradas y la trama de relaciones lleva al trabajador social a pensar en términos de composición y de proceso, de visibilidad de las condiciones y cuestionamiento de los discursos. "La construcción del objeto de trabajo implica la reformulación de la demanda en el desplazamiento de la mirada hacia los sujetos en medio de relaciones, en contextos, en situaciones; los espacios, las formas de enseñanza, la organización escolar misma" (MEN, 2014, p. 29).

Es por esto que se hace necesario pensar a los equipos de orientación en las escuelas en términos de mediadores lingüísticos, en el entremedio, entre las alteridades, en búsqueda de los consensos / disensos / rupturas / crisis, de incentivar a que cada uno sienta la necesidad de comunicarse con el otro. En este espacio, pensar la intertextualidad como la materialidad de la trama en la que interviene el trabajador social en el seno de los equipos de orientación escolar, presenta la posición potencial del "traductor": acercando posiciones, negociando espacios, estructurando discursos que le permitan leer la realidad a todos y cada uno de los sujetos con los que se interrelaciona.

\section{Conclusión}

Pensar la intervención de los equipos de orientación escolar en el marco de la construcción adultocéntrica que la sociedad moderna realiza sobre la juventud y de la escuela como un mecanismo para reproducirla, nos da la posibilidad no solo de caracterizar y enmarcar su trabajo en la trama de relaciones y poderes, sino también nos plantea la 
posibilidad de un trabajo crítico, que conlleve la búsqueda de existencias auténticas en términos de Heidegger.

En esta línea argumental, su horizonte de intervención se recorta en la potenciación del capital participativo orientado a la construcción ciudadana, entendiendo por tal el pleno desarrollo de la libertad personal, la autodeterminación, el ejercicio responsable de derechos, lo que significa poner en juego la capacidad de elección, y ello implica la posibilidad de decidir. Entiendo por tal la idea de una identidad histórica, social, teórica y políticamente construida. (Saibene, 2007, p. 9)

Para esto es necesario que los y las trabajadores sociales que ejercen funciones en las escuelas secundarias puedan incluir en sus prácticas un fuerte trabajo de desnaturalización de los discursos institucionales, profesionales, normativos, del cotidiano, y preguntarse por los supuestos que los sostienen. Asimismo, pensar en las prácticas profesionales sostenidas por estos discursos y por las concepciones adultocéntricas sobre las juventudes, para poder transitar hacia una consideración del otro como sujeto inédito, único y portador de derechos; y no como un sujeto pre armado por los discursos hegemónicos.

Sin embargo, y también a partir de esta línea de pensamiento, se entiende que para poder actuar críticamente es necesario conocer, comprender y dar discusiones a nivel conceptual. Dar batallas que son a nivel de la formación de los discursos dominantes. Para eso es necesario que los trabajadores sociales, como parte del colectivo profesional que conforman los equipos de orientación escolar, podamos estar preparados para enfrentar esta lid, como decía José Paulo Netto, con competencia técnica, política y teórica.

\section{Referencias bibliográficas}

Beck, U. (1997). Teoría de la sociedad de riesgo. En J. Beriain (Comp.), Las consecuencias perversas de la modernidad. Modernidad, contingencia y riesgo (pp. 201-222). Buenos Aires: Amorrortu.

Beck, U, Giddens, A, y Lach, S. (1997). La modernización reflexiva. Política, tradición y estética en el orden social moderno. Buenos Aires: Alianza.

Bourdieu P. (1990). La juventud no es más que una palabra. En Sociología y Cultura. Grijalbo/CNCA, (Los noventa): México.

Castell, R. (1995) La metamorfosis de la cuestión social. México: Paidós. 
Castro, G. (2005). Los jóvenes y la vida cotidiana: elementos y significados de su construcción. Espacio Abierto, 14(1), enero-marzo, 7-23. Disponible en http://www.redalyc.org/articulo.oa?ld=12214101

Castro Gómez, S. (2007). Michel Foucault y la colonialidad del poder. Tabula Rasa (6), 153173.

Castro Gómez, S. (2010). Historia de la gubernamentalidad. Razón de Estado, liberalismo y neoliberalismo en Michel Foucault. Bogotá: Siglo del Hombre / Pontificia Universidad Javeriana /Universidad Santo Tomás.

Cavarozzi, M. (1999). El modelo latinoamericano: su crisis y la génesis de un espacio continental. En M. A. Garreton (Comp.), América Latina: un espacio cultural en un mundo globalizado (pp. 120-139). Bogotá: Andrés Bello.

Chávez, M. (2005). Juventud Negada y Negativizada. Última Década. (23), diciembre, 9-32.

Chávez, M. (2006). Investigaciones sobre juventudes en Argentina: estado del arte en ciencias sociales. Informe para el Proyecto: Estudio Nacional sobre Juventud en la Argentina. Dirigido por Eleonor Faur. IDAES-UNSAM-DINAJU. Buenos Aires. (trabajo no publicado)

Dagnino, E. (1994). Os movimentos sociais e a emergencia de una nova nocao de cidadania. En E. Dagnino (Ed.), Anos 90 - polica e sociedade no Brasil (pp. 103-108). São Paulo: Brasiliense.

Dávila, O. (1998). Exclusión social y juventud popular. Última Década, (8), marzo, 1-19.

Deleuze, G. (2005). Posdata sobre sociedades de control. En C. Ferrer (Comp.), Lenguaje libertario II. Antología del pensamiento anarquista contemporáneo (pp. 115-121). La Plata: Terramar,

Del Río Lugo, N. (2014). Ciudadanía y participación: los nuevos nichos de acción de los jóvenes mexicanos. En G. Tonon (Comp.), Escenarios Cotidianos y Calidad de Vida de Niños, niñas y jóvenes en América Latina. Anales de la IX Conferencia Internacional de la Red Latinoamericana y del Caribe de Childwatch International Research Network 2013, Año 2 (4), setiembre. Colección UNI-COM. Disponible en: http://www.sociales.unlz.edu.ar/unicom/colecionunicom/A2N4-Escenario.pdf (última consulta 10/05/2016)

Erikson, E. (1977). Identidad, juventud y crisis. Buenos Aires: Paidós.

Foucault, M. (1992). Microfísica de poder. Madrid: La Piqueta.

Foucault, M (1996). Vigilar y castigar. Nacimiento de la prisión. México: Siglo XXI.

Foucault, M. (1999). El orden del discurso. Barcelona: Tusquets. 
Foucault, M. (2009). Historia de la sexualidad I. La voluntad de saber. (2ed.) México: Siglo XXI.

Foucault, M. (2015). El sujeto y el poder. En J. Toro (Ed.), Pensamiento y experimentación (pp. 31-52). Bogotá: Carpe Diem.

Giddens, A. (1994). Consecuencias de la modernidad. Barcelona: Alianza.

Gonzalez saibene, A. (2007). El objeto de intervención profesional: un mito en Trabajo Social. Rosario: Fundación Futuro Solidario.

Habermas, J. (1987). Teoría y Praxis, Madrid: Tecnos.

Heidegger, M. (2006). El Ser y el tiempo. Buenos Aires: Fondo de Cultura Económica.

Kamal, C. (2014). Foucault: Pensar mirando hacia Irán... Res Pública. Revista de Historia de las Ideas Políticas, 17(1) 181-197. Disponible en http://dx.doi.org/10.5209/rev_RPUB.2014.v17.n1.45562

Kessler, G. (2004). Sociología del delito amateur. Buenos Aires: Paidós.

Laval, C. y Dardot, P. (2012). La nueva razón del mundo. Barcelona: Gedisa.

Luhmann, N. (1998). Sistemas sociales: lineamientos para una teoría general (Vol. 15). Barcelona: Anthropos.

Margulis, M. y Urresti, M. (1998). La construcción social de la condición de juventud. En H. Cubides Cipagauta; M. C. Laverde Toscano, C. E. Valderrama (Eds.), “Viviendo a toda": jóvenes, territorios culturales y nuevas sensibilidades (pp. 3-21). Santa Fe de Bogotá: Siglo del Hombre Editores.

Netto, J. P. (1998). Opciones hacia el año 2000: un servicio social domesticado o crítico. En Vy VI Jornadas Municipales de Trabajo Social. Buenos Aires: APSS.

Reguillo, R. (2006). En América hay un agotamiento institucional. En Página 12, Buenos Aires. 04/09/06.

Robles, F. (1999). Los sujetos y la cotidianeidad. Elementos para una microsociología de lo contemporáneo. Concepción: Editorial Sociedad Hoy.

Souza Santos, B. (1998). De la mano de Alicia: lo social y lo político en la modernidad. Bogotá: Siglo del Hombre Editores.

Sennett, R. (1978). El declive del hombre público. Barcelona: Península.

Sennett, R. (1983). La Autoridad. Alianza: Madrid.

Vásquez, J. (2011). Jóvenes en la sociedad del miedo: mirada sobre medios, miedos y jóvenes en Ecuador. Revista Latinoamericana de Derechos Humanos, 22(2), 171184. 
Vásquez, J. (2013). Adultocentrismo y juventud. Aproximaciones foucaulteanas. Sophia, Colección de Filosofía de la educación, 15, 215-234.

\section{Otros documentos consultados:}

Balbi, J. (Coord.) (2005). Informe sobre desarrollo humano en la provincia de Buenos Aires 2004-2005. Buenos Aires: Fundación Banco de Provincia de Buenos Aires.

Chávez, M. (2004). La juventud en la escuela. Cuadernillo para capacitación. Dirección de Psicología y Orientación Social Escolar. La Plata: Dir. Gral. de Cultura y Educación, La Plata, Provincia de Buenos Aires, 27 páginas.

Ministerio de Educación de la Nación (2014). Los equipos de orientación en el sistema educativo. La dimensión institucional de la intervención. Buenos Aires: Ministerio de Educación de la Nación. 